\title{
An exploration of NHS clinical staff perceptions of changes to clinical products and their procurement
}

\author{
Clare Donohoe
}

Citation

Donohoe C (2018) An exploration of NHS clinical staff perceptions of changes to clinical products and their procurement. Nursing Management. doi: 10.7748/nm.2018.e1746

Peer review

This article has been subject to external double-blind peer review and has been checked for plagiarism using automated software

Correspondence

clare.donohoe@nhs.net

Conflict of interest

None declared

Accepted

26 October 2018

Published online

December 2018

\section{Abstract}

The NHS in England is working to reduce variation and waste in the use of clinical products, which requires collaboration between those directly involved in procurement and clinical staff. The procurement process is becoming centralised and standardised, and the involvement of, and consultation with, clinical staff is vital to avoid compromising patient care and safety.

This article reports the results of a survey of clinical staff and clinical procurement specialist nurses undertaken as part of a master's degree. Its aim was to capture staff experiences and perceptions of changes to clinical products.

\section{Author details}

Clare Donohoe, clinical procurement specialist nurse, Partners Procurement Service, Whittington Health NHS Trust, London, England

\section{Keywords}

cost effectiveness, economic assessment, management, quality assurance, resource management, supplies

Key points

- The clinical product change process in the NHS is complex, influenced by several factors and not always underpinned by clinical evidence or best practice

- The procurement process is changing; clinical product selection is increasingly being managed centrally

- It is essential that clinical staff engage with and discuss these changes, or they may be imposed

- Clinicians'voices are vital in ensuring that patient care is not compromised, and that decisions on clinical product selection and availability are not based only on cost

\section{Introduction}

The NHS in England seeks to work more efficiently and reduce variation and waste in the use of clinical products. The Carter Report (Department of Health (DH) 2016) on unwarranted variation in productivity and efficiency in the NHS advocates 'greater collaboration, cooperation and economies of scale' and underpins the 
new procurement model, the Future Operating Model (FOM) (DH 2017). The FOM is designed to change how clinical products are procured as trusts face increasing financial pressure and reconfiguration of services. Involvement of, and consultation with, the clinical staff who use these products are vital to ensure that quality of care and patient safety are not compromised.

The role of clinical procurement specialist nurses (CPSNs) involves changes to, and rationalisation and standardisation of, clinical consumable products, which are ward-based consumables that range from basic dressing swabs to more clinically specialist products, such as intravenous cannulae and therapy devices, for which varying degrees of knowledge and training are required.

Reasons for changing clinical products are varied and complex, for example to save money, to improve safety or quality and to address supply issues, and can be planned or unplanned. Success or failure is dictated by identifying products and communicating and working with nurses and other clinical staff to introduce them into practice.

The Effective Clinical and Financial Engagement guide for the NHS (DH 2013) acknowledges that, while finance managers 'have a critical role to play' in engaging clinicians in finance decisions, nurses and other clinicians are the ones who commit NHS resources and need 'a greater understanding of the financial consequences of their actions'.

However, engaging clinical staff in procurement can be challenging and it is often regarded as a low priority due to clinical staff's focus on clinical priorities, service delivery and staffing. Engagement can also be affected by resistance to change, organisational culture and structure, personalities, decision-making, aversion to risk and complex governance processes (Montgomery and Schneller 2007). Improving clinical engagement therefore requires good relationships between clinicians and procurement departments.

As part of my master's degree, I undertook a service evaluation project across the four NHS care providers where I am senior CPSN in an NHS partners procurement service (PPS). The aims of the project, relevant to this article, included: to explore staff knowledge, experience and perception of clinical product change and reasons for change. The longer-term aim was to use the findings to form an action plan to improve clinical engagement in the four trusts. This article describes one part of the project, a survey of staff perceptions and experiences of the product change process.

\section{Background}

National initiatives to increase awareness of and engagement in clinical product change include the Royal College of Nursing (RCN) Small Changes, Big Differences campaign (RCN 2015). The campaign highlighted the importance of involving nursing staff in product change to ensure that patient care is not compromised and advocated the reduction of clinical product variation to improve patient safety outcomes. According to the RCN (2015), standardisation would enable training to be put in place during product changes, increase familiarity with the products irrespective of department and would be more cost effective.

A DH national clinical evaluation team (CET), comprising registered nurses with a clinical procurement or other clinical background such as infection prevention or tissue viability, was set up in 2016 to conduct independent reviews of the specifications of ward-based clinical consumable products. This was to underpin a new procurement programme called the Nationally Contracted Products (NCP) programme (DH 2017). As the title suggests, clinical products will be contracted nationally and, once they are deemed clinically acceptable, the DH and NHS Improvement (NHSI) expect trusts in England to adopt them, reducing clinician preference and variation. For this to be successful, relationships with clinicians need to be managed locally. Good communication is essential to reassure clinical staff that the products selected are fit for purpose and to inform staff about how to raise concerns and give feedback.

It is important to ensure clinical staff know that, if they do not express a view about products for patient treatment and care at this point, changes by bodies such as NHSI, which are more removed from patient care, will be imposed on them. 


\section{Literature review summary}

A literature review was undertaken to identify and explore current practice and to establish if there are similar studies to this one. Databases searched included Ebsco, Proquest and Athens and relevant sources of information were identified in procurement and clinical journals, health department websites and various organisations' reports. Based on the search terms 'procurement', 'clinical engagement' and 'clinical products', procurement journals provided more relevant information than clinical and nursing journals, which yielded little material.

Although no literature that directly relates to the aims of the project was generated, some relevant issues were identified. For example, Pierpoint (2012) discussed how, in his view, the role of the NHS Supply Chain procurement specialists is pivotal to understanding the needs and requirements of clinicians, while Brooke (2012) identified that clinical engagement is a central component of the CPSN role. Meanwhile, Blakemore (2015) described the benefits and value of nursing staff involvement in clinical procurement decisions but identified variation across organisations.

Meehan et al (2016) explored the barriers to collaborative procurement and resistance to change in five UK public authorities in the emergency care sector and found that stakeholders legitimise resistance to change on the pretext of a reduction in control over product choice. The authors also found that for clinicians, safety outweighed commercial or cost implications and national directives, that there was a persistent belief in the organisations studied that 'regional collaborative procurement (is) too risky to implement' and that there is a culture in which stakeholders regard procurement primarily in the context of 'ensuring process compliance'.

Meehan et al (2016) also discussed factors such as the emotive nature of procurement and procurement strategies, including protectionism to resist change. They concluded that successful implementation of collaborative procurement strategies rely on identifying the forces and barriers that cause stakeholders to resist collaboration, but warned that such barriers are 'many, complex and deeply engrained', requiring 'iterative, immersive and stakeholder-engaged' solutions.

These issues, resistance to change, loss of clinician autonomy, safety considerations and best technical specification at odds with national directives, reflect my own experiences and that of other CPSNs. However, the main topic of this project is not examined in detail in the literature and warrants further investigation, particularly in the context of accelerated clinical product change.

\section{Survey}

An online staff survey questionnaire using SurveyMonkey was designed to explore the experiences and perceptions of clinical staff in relation to clinical product change and to improve communication and engagement with and involvement of clinical staff in the clinical product selection and change process.

The survey was used in two ways: the first involved a focus on PPS-supported clinical staff at the four trusts (survey 1), the second a focus on members of the CPSN network peer group, a national network of nurses who work in a CPSN role in England and Scotland (survey 2).

The primary survey (survey 1) comprised ten questions with space for free text. The question topics and related themes are shown in Table 1. The CPSN network, for survey 2, was asked to select the response they thought would most likely be chosen by clinical staff if the same questions were asked in their organisations. The responses were compared.

\section{Table 1. Research questions and relevant themes}

\section{Question topic}

1. Information about the respondent: organisation, clinical setting and clinical discipline

2. Name of the procurement shared-service trust where the respondent works

3. Information about clinical product change

\section{Theme}

Context 
4. Opportunity to ask questions and receive training

Experience and

perception of

5. Staff participation in the product change process

product change

6. Adequacy of information of product change and awareness of who to contact for support or if questions or concerns arise

7. How important it is for staff to know when clinical products are changing

Importance of

knowing products

8. Reasons for clinical product change

are changing

9. Simple self-assessment of cost of clinical products

Cost

10. Acceptability of clinical product change based on the same change made satisfactorily elsewhere

Acceptability of product change

A definition of ward-based consumables was given in the introduction and background section of the survey and staff were asked to use the examples of produce changes when answering the questions to help put their responses into context. To avoid ambiguity, a 'depends on the product' option was incorporated. The aim was to encourage staff to complete all sections. Mainly open questions were used. Feedback from a pilot version of the survey was used to refine the questions.

\section{Findings}

A total of 135 staff from the four NHS trusts participated in survey 1 and 21 CPSNs completed survey 2 based on how they expected clinical staff would respond. The findings are outlined below and linked to the themes listed in Box 1. Survey 1 respondents' roles are shown in Figure 1.

Figure 1. Respondents' roles: survey 1, question 1

\section{Experience and perception}

Questions relating to topics 3, 4, 5 and 6 were designed to determine staff perception of information about product change, and responses were used to establish a baseline of how staff felt.

Figure 2 shows a comparison of responses to question 3, which was a 'yes/no' response with the 'yes' options listing the main routes through which product changes are communicated to staff.

\section{Figure 2. Being informed about changes to clinical products: comparison of responses to question 3 in surveys 1 and 2}

Question 4 asked about the opportunities for staff to ask questions about and receive training in the use of new products before changes are made and responses were broadly similar in both survey groups. Only about one third (31\%) of staff in survey 1 agreed there had been such opportunities, which could affect competency and has patient safety implications.

Question 5 related to staff involvement in decisions about product change and opportunities to give their view. The 'yes' options enabled respondents to provide further information on how they were involved (Figure 3).

\section{Figure 3. Staff involvement in product change decisions: comparison of responses to question 5 in surveys 1 and 2}

The high 'no' response in survey 1 (Figure 3) was surprising as it was expected that staff involvement would be greater and reflect the work undertaken in the PPS. However, this finding correlates with responses to question 6 , which examined adequacy of information at the time of product change (Figure 4).

Responses in survey 1 indicate that clinical staff in the PPS trusts felt less informed than their counterparts in trusts with a CPSN. The variation in the CPSN role warrants consideration as some cover only one trust while others cover several, and the role may be resourced differently, with some, for example, being supported by a team 
of nurses. It was uncertain whether question 6 could be subjective as CPSN respondents (survey 2) might like to think that staff in their trusts are well informed.

\section{Figure 4. Adequacy of information at the time of product change: comparison of responses to question 6 in surveys 1 and 2}

The free-text section of survey 1 contained emotive language, and comments suggested that changing clinical products is often perceived as negative. For example, one respondent said 'Due to cost pressures, sub-par products are being used', while the word 'inferior' was used frequently. Staff reported finding out that products were no longer available only when they went to order them, which could have operational or clinical implications.

\section{Importance of knowing products are changing}

Survey 1 (Figure 5) shows that three quarters of respondents apportion a high level of importance to knowing about changes to clinical products compared to the far lower value given by CPSNs (survey 2). This questions whether trust procurement and finance departments and CPSNs correctly estimate the importance of providing information to clinical staff and whether staff communicate how important they feel this is, responding instead with antipathy or resignation. A further point to consider is whether it is simply change itself that engenders negative feelings among staff.

\section{Figure 5. Importance of knowing that products are changing: comparison of responses to question 7 in surveys 1 and 2}

Question 8 focused on staff understanding of why clinical products change and a range of options was given to capture as many reasons as possible. More than one reason could be selected, and respondents could select all options if relevant. In both surveys, a high percentage of staff cited financial reasons and supply disruption for product changes, which were anticipated responses. Staff preference was the lowest reason given, which implies that staff believe clinical product changes are more financially than clinically driven. Table 2 shows a comparison of responses to staff understanding of change in clinical products.

\begin{tabular}{l|l|l|l|l|l|}
$\begin{array}{l}\text { Table 2. Comparison of responses to question } \mathbf{8} \text { in } \\
\text { surveys } \mathbf{1} \text { and } \mathbf{2}\end{array}$ & $\begin{array}{l}\text { Survey } \mathbf{1} \\
\text { Reason for response }\end{array}$ & Survey $\mathbf{2}$ \\
\hline \% & $\boldsymbol{n}$ & $\%$ & $\boldsymbol{n}$ \\
\hline Supply issue or product unavailable & 66 & 89 & 76 & 16 \\
\hline Product recall or quality issue & 52 & 70 & 19 & 4 \\
\hline To save money & 96 & 128 & 100 & 21 \\
\hline New product ensures better patient outcomes & 49 & 66 & 62 & 13 \\
\hline Staff preference & 10 & 13 & 19 & 4 \\
\hline Supplier of new product advises it is better & 20 & 27 & 24 & 5 \\
\hline Other & 6 & 8 & 10 & 2 \\
\hline
\end{tabular}

\section{Cost}

Question 9 used a Likert scale of between 0 and 100 to gauge staff knowledge about the cost of clinical products and respondents were asked to self-assess their level of knowledge. Survey 1 revealed an average score of 45 compared with an average score of 36 in survey 2 . Although there was wide variation, overall the average score in either survey was lower than $50 \%$, which suggests that staff awareness about cost is poor and that work to promote product cost awareness should be considered. 
There are limitations to the accuracy of this method of measurement. Bowling (2014) highlighted that respondents commonly opt for a 'middle response category' to avoid a commitment or decision. Due to time constraints, a detailed data analysis to exclude outliers at extremities of either end of the scale was not undertaken.

This question was deliberately inserted at a later stage of the survey to prevent staff from believing the motive for the survey was to save money and that their views on other issues would not be considered. However, cost was raised by respondents in the free-text section and included comments such as 'Other Trusts may have made a poor choice, but it was forced on them because it was cheaper'. Analysis of the free-text data, using the number of references made to cost through the SPSS and NVIVO tools, suggests that, while respondents accepted that some products could be 'generic' and acknowledged the effect of marketing, they strongly believed that 'cheaper' products were inferior.

\section{Acceptability of change}

Question 10 addressed the acceptability of a clinical product change based on the same change having been made satisfactorily in other trusts. CPSNs use evidence such as this, that a clinical product is used effectively in other PPS trusts or in the wider NHS with no evidence of harm, as a basis for recommending a change. Responses to survey 1 (Figure 6) indicate that, despite this, clinical staff require additional reassurance and to test products for themselves. This suggests that it cannot be assumed that clinicians will accept changes to clinical products based on their successful use in other care providers.

\section{Figure 6. Acceptance of product change based on uptake by other trusts: comparison of responses to question 10 in surveys 1 and 2}

\section{Limitations}

The survey was conducted corresponded during a period of intense activity of project implementation with accompanying communications through existing trust routes advising staff of proposed product changes. The use of a reflective diary gave cause to consider whether ongoing intense and concurrent project activity could potentially skew the survey responses. If staff believed there was good engagement based on recent experience, it would be unclear whether their responses were representative of the wider group.

The 135 clinicians who completed survey 1 represent a small sample of staff in all trusts, which collectively employ tens of thousands of such staff. Variation in routes of dissemination of the staff survey was also a limitation in that not all trusts involved could disseminate it centrally.

Supportive members of staff who had attended trust clinical forums or had been involved in previous product change projects were identified and acted as 'champions' by disseminating the survey in their areas. Subsequently, responses were influenced by what is in place in those clusters.

The author kept a reflective journal throughout the project to provide trustworthiness and validity. The log also serves to manage and question any bias that could steer the enquiry.

\section{Developments based on findings}

\section{Communication}

The way procurement teams communicate product changes to the trusts involved has been reviewed based on feedback from the survey. Timeframes to communicate the introduction of new products may need to be extended to ensure that all groups affected are adequately informed. However, this must be balanced with the demands of the CPSN role to implement change projects to agreed timescales.

Discussion has taken place with the CPSN network to determine why communication with clinical staff is allegedly better in their trusts, and what learning could be used to improve communication with clinical staff.

\section{Raising awareness}


Staff awareness of the cost of clinical products has been discussed by trust working groups. Tools such as a 'traffic light' system of presenting clinical product cost at ward level have been designed but had not been implemented in the trusts at the time of writing. The lack of knowledge but expression of interest in learning about this aspect of procurement cited by respondents makes a case for further discussion with senior nursing staff about awareness campaigns or local or trust-wide pilot projects.

\section{Trust strategy and clinical concerns}

The ability to implement savings by changing to more cost-effective clinical products 'at pace' is directly affected by the factors highlighted by responses to question 10 concerning reluctance to change to products used successfully in other PPS trusts. Savings targets are increasingly ambitious and require a strategy to address and manage better clinicians' concerns and their need to 'test for themselves', which will increase time to implementation and savings realisation.

This question also highlights an apparent lack of trust by clinicians in the clinical product procurement process and has implications for CPSNs in addressing these concerns.

Comparing different cultures and exploring reasons for resistance across the trusts involved is beyond the scope of this project. However, it does capture how the power in the different stakeholder groups varies. For example, one respondent noted: 'Medical device/products ie, doctors are driven by consultants depending on their preference, skill and speciality. Whereas, non-medical ie nursing products are driven by cost.'

\section{Action plan}

An action plan for implementation across the four trusts is shown in Table 2.

\section{Table 2. Outcomes and action plan}

\section{Outcome}

1. Working differently from current practice

\section{Communicating survey findings to internal and external stakeholders}

Exploring changes in practices and methods of information dissemination to clinical staff in line with practices in other NHS trusts where they have been shown to be more effective

4. Preparing and presenting an action plan to improve engagement to employer and relevant trust stakeholders

5. Depending on feedback and outcomes, comparing information across trusts

\section{Action}

Apply knowledge and skills gained to practice

Present to:

»Medical surgical team and partners procurement service (PPS) director

》 Trust stakeholders, as advised by director

»Clinical procurement specialist nurse (CPSN) network meeting

»Other external stakeholders, such as the Royal College of Nursing and Department of Health

Discussion with clinical network about outcomes of an adapted staff survey 1 completed by staff in other trusts and of peer group survey 2 in context of other CPSN settings

As for objective 2

Analysis of survey feedback from trusts or staff groups, as defined and requested by PPS or trust,

\section{How}

Dealing with stakeholders, such as senior nurses and clinicians

Use of presentation software and CPSN portal

Discussion of best practice and good clinical

engagement with CPSN members at a meeting and through the CPSN portal

As for objective 2

Use of spreadsheet software and data analysis
In progress

Donohoe

\section{Timescale}

Completed by

In progress

Donohoe

To be advised Donohoe

From February Donohoe and 2018 CPSN network

\section{From February Donohoe} 2018 
and presenting the result to tools, with help of trust data

relevant trust forums as requested analyst staff if appropriate

\section{Conclusion}

The findings of this survey correlate with some of the points raised in the literature review including the variability in clinical staff involvement in procurement (Blakemore 2015). The free-text section provided some interesting insights; for example, one respondent said: 'Not all trusts are the same and just because one trust has relented or accepted inferior products, we should not need to follow suit. The clinicians should get final say in changes. Our medical licences are on the line each day and we should not have to use unsuitable or unpreferred [sic] equipment just to save money. A critical incident contributed to by unfamiliar or unpreferred [sic] equipment soon makes these savings a false economy.' This illustrates the strength of feeling about proposed changes to clinical products. Other respondents, however, were more positive and expressed a desire to know more about the cost of clinical products.

This project illustrates that the clinical product change process in the NHS is complex, influenced by many factors and not always underpinned by clinical evidence or best practice. As the procurement landscape changes, with directives on clinical product selection dictated from the centre, standardisation and rationalisation with attendant reduction of product choice, it is essential that clinical staff engage with the process and have their say, or changes will be imposed. Clinicians' voices are vital in ensuring that patient care is not compromised and that decisions on clinical product selection and availability are not based only on cost. Nurses are integral to this process.

\section{Recommendations for practice}

»Do you have a CPSN or clinical specialist in your organisation? The number of CPSNs has increased substantially as trusts recognise the value of this role.

» Get to know your procurement staff. They can support you in different ways, such as raising awareness of cost, labelling storeroom drawers with prices and helping to manage stock.

"Speak up. Clinicians are best placed to see the issues, such as there being excess stock or waste.

» Get involved in product selection through product forums or clinical product evaluations. Doing so can also offer staff development opportunities.

\section{References}

Blakemore S (2015) Buying into a greater product procurement role for nurses. Nursing Management. 22, 8, 8-9.

Bowling A (2014) Research Methods in Health Investigating Health and Health Services. Fourth edition. Open University Press, Berkshire.

Brooke C (2012) Clinical procurement specialist: a personal perspective. Journal of Perioperative Practice. 22, 5, 4-5.

Department of Health (2013) Effective Clinical and Financial Engagement. A Best Practice Guide for the NHS.

assets.publishing.service.gov.uk/government/uploads/system/uploads/attachment_data/file/255655/effective_clinical_financial_engagement.pd f (Last accessed: 27 November 2018.)

Department of Health (2016) Operational Productivity and Performance in English NHS Acute Hospitals: Unwarranted Variations. An Independent Report for the Department of Health by Lord Carter of Coles. assets.publishing.service.gov.uk/government/uploads/system/uploads/attachment_data/file/499229/Operational_productivity_A.pdf (Last accessed: 27 November 2018.)

Department of Health (2017) NHS Transformation Programme Future Operating Model (FOM) Handbook. Issue 1: October 2017. DH, London. URL: https://www.supplychain.nhs.uk/sccl/ /media/Files/News/FOM_HANDBOOK\%200ct\%202017.ashx

Meehan J, Ludbrook M, Mason C (2016) Collaborative public procurement: Institutional explanations of legitimised resistance. Journal of Purchasing and Supply Management. 22, 160-170. 
Montgomery K, Schneller E (2007) Hospitals' strategies for orchestrating selection of physician preference items. Milbank Quarterly. 85, 2, 307-335.

Pierpoint D (2012) Inside clinical procurement - what you didn't know. Journal of Perioperative Practice. 22, 9, 5-7.

Royal College of Nursing (2015) Small Changes, Big Differences. Involving Nursing Staff in the Procurement of Clinical Supplies. RCN, London. URL: https://www.rcn.org.uk/small-changes

Figure I. Respondents' roles: survey I, question I

1. About you and your role: please choose one of the below and state your area of practice

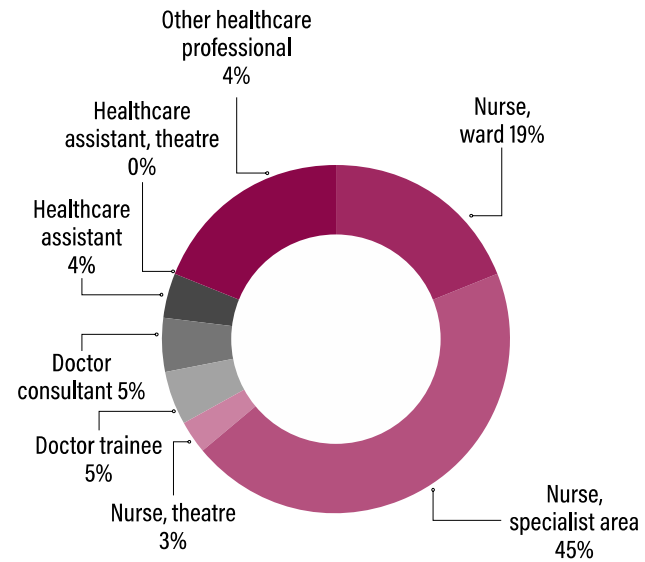

Figure 2. Being informed about changes to clinical products: comparison of responses to question 3 in surveys I and 2

3. When clinical products have changed were you informed and if so how?

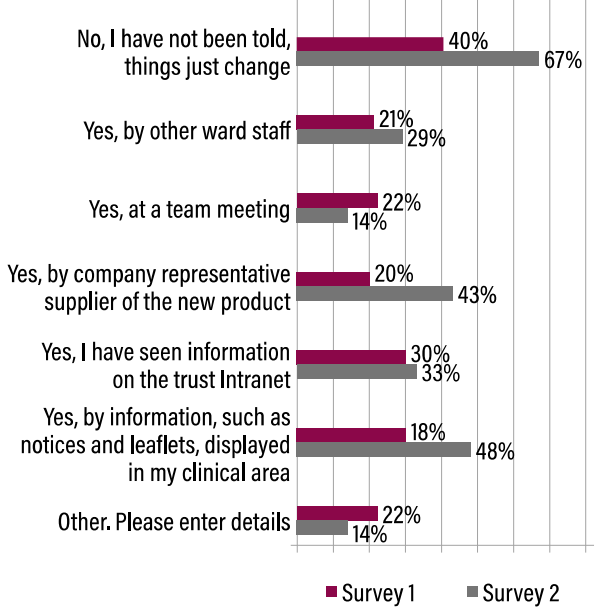


Figure 3. Staff involvement in product change decisions: comparison of responses to question 5 in surveys I and 2

5. Staff involvement in product change decisions: could staff give their view?

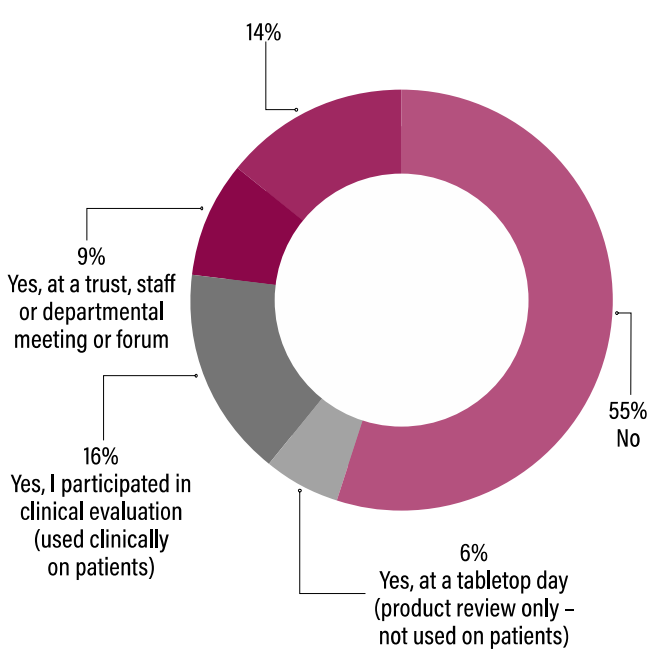

Survey 1

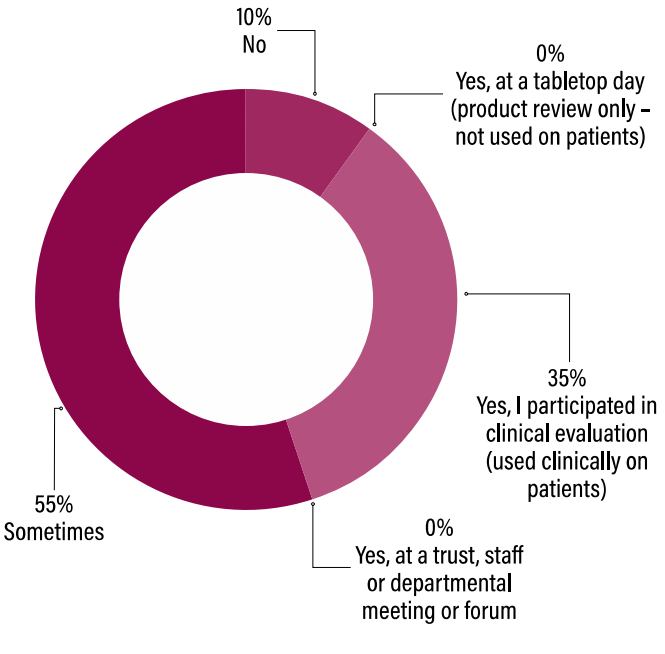

Survey 2

Figure 4. Adequacy of information at the time of product change: comparison of responses to question 6 in surveys I and 2

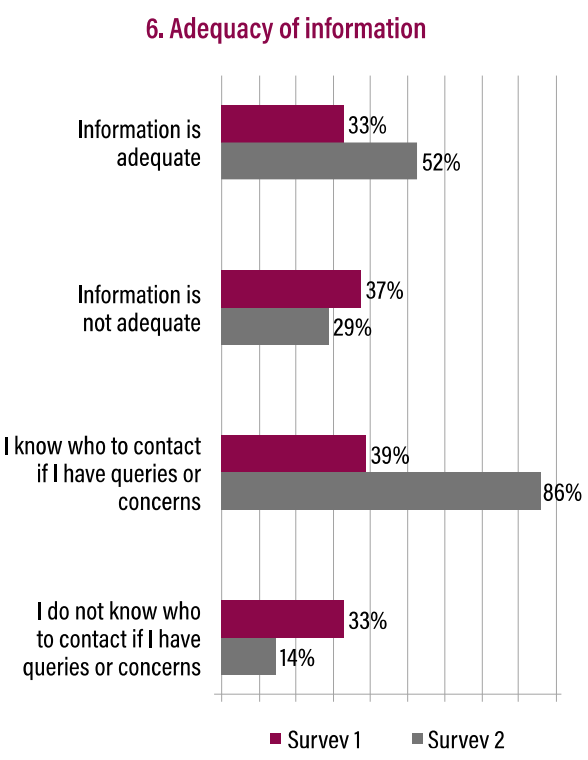


Figure 5. Importance of knowing that products are changing: comparison of responses to question 7 in surveys I and 2

7. How important is it to you to know that products are changing?

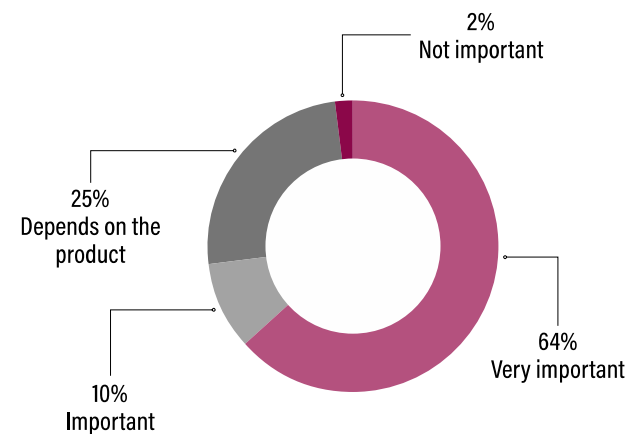

Survey 1

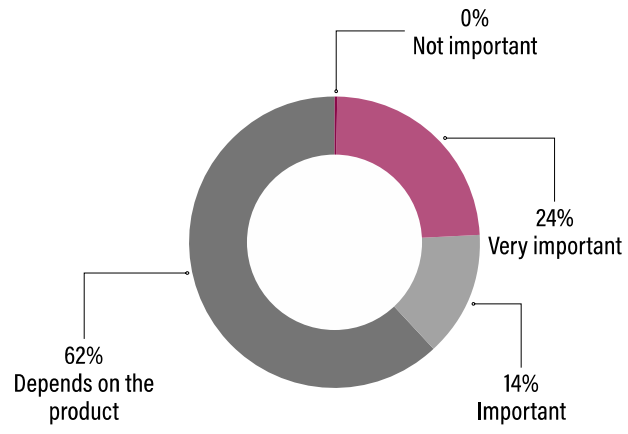

Survey 2

Figure 6. Acceptance of product change based on uptake by other trusts: comparison of responses to question 10 in surveys I and 2

10. Acceptance of product change based on feedback and uptake by other trusts

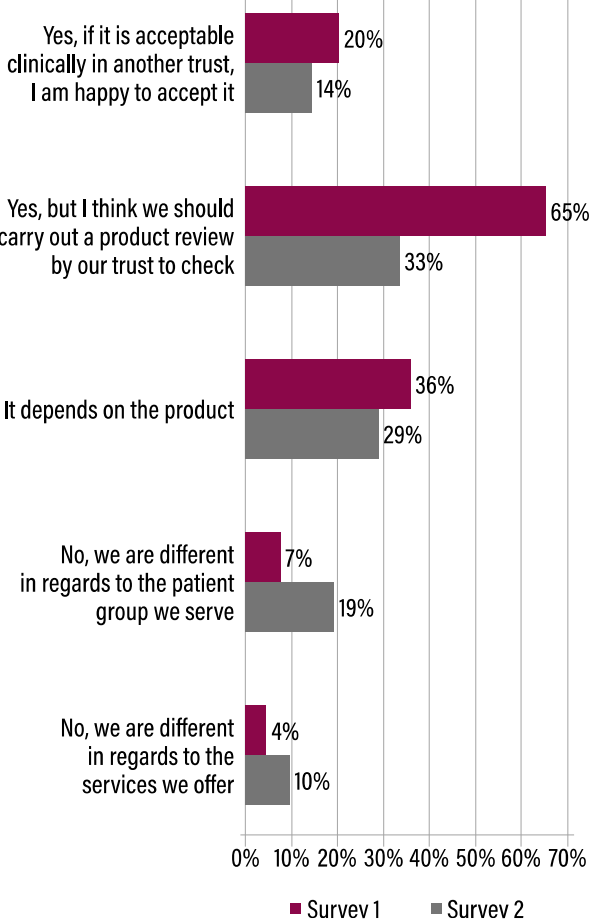

